short-wave diathermy) and advice, or sham exercise and sham advice. The treatment phase was 6 weeks (12 exercise or sham exercise sessions, 3 advice or sham advice sessions). Follow-up to assess the three main outcomes (pain, global perceived effect and functional ability) was attended by 231 participants at both 6 weeks and 12 months.

At 6 weeks' follow up, either advice or exercise was slightly more effective than placebo, but the greatest improvement in all three outcomes was seen when the interventions were combined. The effects of the individual interventions were no longer significant at 12 months; however, the combined intervention resulted in continued improvement in function. The authors suggest that combination treatment with exercise and advice should be considered for patients with subacute low back pain.

Original article Pengel LHM et al. (2007) Physiotherapistdirected exercise, advice, or both for subacute low back pain. Ann Intern Med 146: 787-796

\section{Hydroxychloroquine use reduces the risk of diabetes in patients with RA}

Hydroxychloroquine, an antimalarial drug, is a safe and inexpensive treatment for rheumatoid arthritis (RA) and other autoimmune diseases. The drug improves insulin secretion and peripheral insulin sensitivity in animal models and has hypoglycemic effects in humans. To investigate the association between use of hydroxychloroquine and the risk of incident diabetes in patients with RA, Wasko and colleagues conducted a prospective, multicenter, observational study of 4,905 adults over a 21.5 year period.

Of the 1,808 adults who had ever taken hydroxychloroquine, 54 developed diabetes during the observation period; in contrast, 171 of the 3,097 who had never taken it developed the disease. After adjustment for diabetes risk factors and other covariates (including BMI, functional status and corticosteroid use), use of hydroxychloroquine was associated with a reduced risk of developing diabetes; risk reduced further with increasing duration of use. The reduction in risk was up to $77 \%$ for patients who had taken the drug for $>4$ years compared with the risk for those who had never taken the drug. The incidence per 1,000 person-years was 5.2 for ever-users versus 8.9 for never-users $(P>0.001)$.

The finding of a duration-related association between use of hydroxychloroquine and reduced risk of diabetes in $\mathrm{RA}$ patients deserves further prospective assessment to determine whether the drug should be considered standard combination therapy for these patients or a preventative therapy for individuals at high risk of developing diabetes, or both.

Original article Wasko MCM et al. (2007)

Hydroxychloroquine and risk of diabetes in patients with rheumatoid arthritis. JAMA 298: 187-193

\section{Some antidepressants are associated with increased rates of bone loss}

Many antidepressants function by inhibiting serotonin transporters in neurons. Serotonin transporters are now known to also be expressed in osteoblasts, osteoclasts and osteocytes. Studies have shown an association between antidepressant use and an increased risk of fractures, and between selective serotonin reuptake inhibitor (SSRI) antidepressant use and decreased bone mineral density (BMD) in older men. Diem et al. have conducted a study to determine whether antidepressant use is associated with increased hip bone loss in elderly women.

Rates of hip bone loss were analyzed for 2,722 women (mean age 78.5 years): patients using only SSRI antidepressants $(n=198)$, patients using only tricyclic antidepressants (TCAs; $n=118$ ), or patients not using antidepressants at all (nonusers; $n=2406$ ) were included in the study. BMD was measured on two occasions separated by an average of 4.9 years. SSRI users exhibited higher rates of hip bone loss than nonusers, even when various measures of health status, functional status and weight change were taken into consideration, or when excluding patients with six or more symptoms of depression (previously associated with lower BMD), as measured by a Geriatric Depression Scale questionnaire. TCA users had similar rates of bone loss to nonusers.

The authors conclude that SSRI use is associated with increased rates of bone loss 